\title{
HYDROGEN DIFFUSION AND STRAIN DISTRIBUTION IN AMORPHOUS Fe-BASED RIBBONS
}

\author{
Mária KLADIVOVÁ, Ján ZIMAN, Ladislav NOVÁK, Mária KOVALAKOVÁ \\ Department of Physics, Faculty of Electrical Engineering and Informatics, \\ Technical University of Košice, Park Komenského 2, 04200 Košice, Slovak Republic, tel. +421 55602 2760, \\ e-mail: ladislav.novak@tuke.sk, jan.ziman@tuke.sk, \\ maria.kovalakova@tuke.sk,maria.kladivova@tuke.sk
}

\begin{abstract}
The paper deals with the study of strain distribution in hydrogenated FeB ribbons prepared by rapid quenching from the melt. Hydrogen atoms introduced during the process of hydrogenation induce internal stresses in the sample. This causes bending of a one side hydrogenated non-fixed ribbon. The ribbon bending reflects hydrogen concentration. When the ribbon is dehydrogenated the ribbon shape is fully restored for the studied $\mathrm{Fe}_{85} \mathrm{~B}_{15}$ sample. A simple theoretical model is proposed to describe dependence of ribbon bending on the hydrogen concentration during process of hydrogenation as well as in the process of spontaneous dehydrogenation. A proposed theoretical model is in a good agreement with experimental results. Its fitting to experimental data provides important information on the local enlargement of the space around introduced hydrogen atoms.
\end{abstract}

Keywords: hydrogenation, concentration, magnetoelastic anisotropy, amorphous ferromagnetic ribbons

\section{INTRODUCTION}

Amorphous ferromagnetic materials prepared by rapid quenching from the melt [1] are very promising materials for technical applications and for this reason they are quite often the subject of basic research. The results reported in this paper follow on the study of hydrogenation of amorphous ferromagnetic ribbons as the method for nondestructive investigation of their mechanical-magnetic properties and their possible modification [2,3,4,5] which has been published recently [6]. In the latter paper a theoretical model for hydrogenation and spontaneous dehydrogenation of ferromagnetic ribbons was proposed. Hydrogen atoms introduced into studied amorphous FeB materials increase internal stress in their structure. The rate of hydrogen diffusion in these materials is not fast enough to produce homogeneous hydrogen distribution in the ribbon cross section [6] and consequently also the stress distribution in the ribbon cross section is not homogeneous. The ribbon bending reflecting this nonhomogeneity in the case of one side hydrogenation process (see Fig. 1) can provide information on the induced stress which depends on the hydrogen concentration. This information is of great importance for the studied Fe-based materials which possess large magnetostriction. In the present paper a simple theoretical model is proposed to describe dependence of ribbon bending on the hydrogen concentration during the process of hydrogenation as well as in the process of spontaneous dehydrogenation. Proposed theoretical model is in good agreement with experimental results. Fitting model equation to experimental data makes it possible to obtain important information of local enlargement of the space close to the introduced hydrogen atom, which can change local magnetic characteristics. The theoretical results obtained are the base for further study of such important magnetic characteristics of studied materials as e.g. magnetoelastic anisotropy constant.

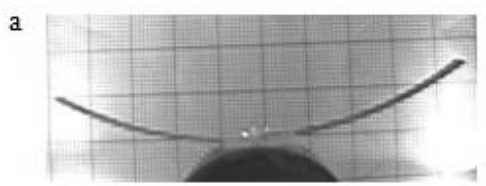

b

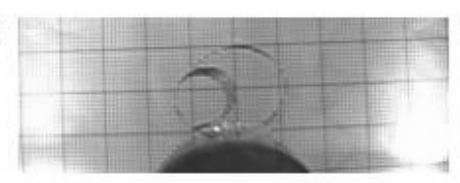

c

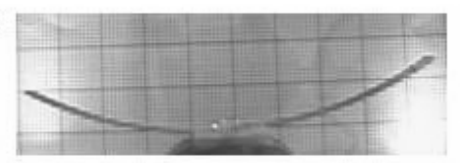

Fig. 1 Bending of hydrogenated sample: (a) - before hydrogenation, (b) - strong hydrogenated, (c) - after dehydrogenation

\section{MODEL}

We consider a model situation in which the length and width of the ribbon are much larger than its thickness $L$, and the material of a ribbon has no affinity to hydrogen. The aim of the following considerations is to study the influence of one side hydrogenation on the bending of a thin amorphous ferromagnetic ribbon and also on strain distribution.

In the ribbon hydrogenated from one side (from the left side in Fig. 2) the hydrogen located in the matrix causes local volume increase which results in an observable isotropic elongation (perpendicular to the $x$ axis, see Fig. 2) of each elementary layer from which the ribbon can be considered to consist of. An elongation in the direction perpendicular to the plane of the sample (in the $x$-axis direction) is negligible small for thin ribbon and 
its influence on the bending of hydrogenated sample in the simple model presented here is neglected. In the case of one-side hydrogenation (in the $x$-axis direction) sample bending is observed as can be seen in Fig. 1.

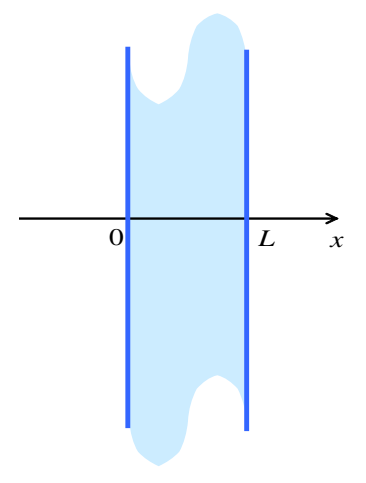

Fig. 2 Sample with a thickness $L$

Due to the isotropic enlargement of the surface of one side of hydrogenated sample the ribbon bends and its surface becomes spherical which reflects successively decreasing hydrogen concentration inside the sample with the distance from the hydrogenated surface [6]. Curvature radius as a function of hydrogen concentration can be obtained from the experiment and can give important information on material constants of a deformed ribbon. Side view of the bent ribbon hydrogenated from the left side (surface $x=0$ ) is schematically depicted in Fig. 3 .

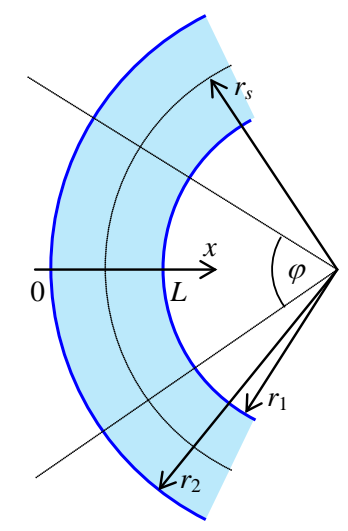

Fig. 3 Side view of the ribbon hydrogenated from the surface $x=0$, where $r_{1}$ and $r_{2}$ are minimum and maximum radii, respectively and $r_{s}$ is a mean curvature radius

Theoretical dependence of a mean curvature radius $r_{s}$ on hydrogen concentration can be obtained in the following way.

The length of middle circular arc $l_{s}$ with radius $r_{s}$ of a deformed (hydrogenated) sample is

$l_{s}=l_{0}+\Delta l$,

where $l_{0}$ is the length of the ribbon before hydrogenation and $\Delta l$ is an elongation of its middle part in hydrogenated sample. The length of an arbitrary arc $l_{r}$ with radius $r$ is

$l_{r}=r \varphi$, where $\varphi$ is its central angle. Difference in arc lengths $l_{s}$ and $l_{r}$ is (see Fig. 3 )

$l_{r}-l_{s}=\left(r-r_{s}\right) \varphi=\Delta r \varphi=\left(\frac{L}{2}-x\right) \varphi$.

Using Eqs. $(3,1)$ the length of $l_{r}$ is

$l_{r}=l_{s}+\Delta r \varphi=l_{0}+\Delta l+\left(\frac{L}{2}-x\right) \varphi$.

The strain $\varepsilon$ (relative elongation) for the arc with radius $r$ can be obtained from Eq. 4)

$\varepsilon=\frac{l_{r}-l_{0}}{l_{0}}=\varepsilon_{0}+\varepsilon_{r}$,

where

$\varepsilon_{0}=\frac{\Delta l}{l_{0}}, \varepsilon_{r}=\frac{(L / 2-x) \phi}{l_{0}}$.

The ribbon bending is caused by the presence of hydrogen inside its structure, therefore the strain has to depend on hydrogen concentration ${ }^{1}$. In the presented model we assume that hydrogen concentration $\phi$ is not very high, so Young's modulus $E$ can be considered as constant. Under this assumption strain $\varepsilon$ can be expressed in the form

$\varepsilon=K \phi+\frac{\sigma}{E}$,

where $\sigma$ is internal stress, coefficient $K$ characterizes a volume which is occupied by hydrogen inside the material- volume expansion due to the presence of hydrogen atoms in the matrix per one hydrogen atom. In the presented model, for not very high concentration, it can also be considered to be constant.

The mean value ${ }^{2}$ of hydrogen concentration $\langle\phi\rangle$ in the sample as well as curvature of the ribbon can be obtained experimentally. Therefore, to confront experimental results with theoretical model, the dependence of the middle radius of curvature of the ribbon on the hydrogen concentration $\langle\phi\rangle$ should be found.

The bent sample is in equilibrium state therefore mean value of internal stress is equal to zero

$\langle\sigma\rangle=0$

Also the mean value of $\varepsilon_{r}$, as follows from Eq. (6), equals to zero,

${ }^{1}$ An influence of gravitational field is negligibly small and will not be taken into account.

${ }^{2}$ All quantities depend only on variable $x$ (see Figs. 1 and 3) or $r$ for the bent sample (see Fig. 3), therefore the mean value of quantity $f$ over the sample volume is $\langle f\rangle=\frac{1}{L} \int_{0}^{L} f(x) \mathrm{d} x$. 


$$
\left\langle\varepsilon_{r}\right\rangle=0
$$

Using Eqs. $(5,7,8,9)$ we obtain

$$
\varepsilon_{0}=K\langle\phi\rangle \text {. }
$$

Finally, using Eqs. $(5,7,10)$, the internal stress $\sigma$ can be expressed as

$$
\frac{\sigma}{E}=K(\langle\phi\rangle-\phi)+\varepsilon_{r} .
$$

In the equilibrium the energy associated with the deformation $\left(\sim \frac{1}{2} \sigma^{2} / E\right)$ is minimal as well as its mean value

$$
\frac{\left\langle\sigma^{2}\right\rangle}{2 E}=\frac{E}{2}\left\langle\left(K(\langle\phi\rangle-\phi)+\varepsilon_{r}\right)^{2}\right\rangle .
$$

Using Eqs. $(9,12)$ the last equation can be rewritten in the form

$$
\frac{\left\langle\sigma^{2}\right\rangle}{2 E}=\frac{E}{2}\left[K^{2}\left\langle\langle\langle\phi\rangle-\phi)^{2}\right\rangle+\left\langle\varepsilon_{r}^{2}\right\rangle-2 K\left\langle\varepsilon_{r} \phi\right\rangle\right] .
$$

The expression for mean values ${ }^{2}$ in the last two terms in Eq. (13) can be found using Eqs. $(1,2,6)$

$$
\begin{aligned}
& \left\langle\varepsilon_{r}^{2}\right\rangle=\frac{1}{12} \frac{l_{s}^{2}}{l_{0}^{2} r_{s}^{2}} L^{2}, \\
& \left\langle\varepsilon_{r} \phi\right\rangle=-\frac{l_{s}}{l_{0} r_{s}}\left(\langle\phi x\rangle-\frac{L}{2}\langle\phi\rangle\right) .
\end{aligned}
$$

Finally, using Eqs. (14,15), Eq. (13) can be rewritten in the form

$$
\frac{\left\langle\sigma^{2}\right\rangle}{2 E}=\frac{E}{2} \cdot u,
$$

where

$$
u=K^{2}\left\langle(\langle\phi\rangle-\phi)^{2}\right\rangle+\frac{1}{12} \frac{l_{s}^{2}}{l_{0}^{2} r_{s}^{2}} L^{2}+2 K \frac{l_{s}}{l_{0} r_{s}}\left(\langle\phi x\rangle-\frac{L}{2}\langle\phi\rangle\right)
$$

As was mentioned above, in the equilibrium the value of deformation energy is minimal and mean radius of curvature $r_{s}$, can be found as a solution of equation

$$
\frac{\mathrm{d}}{\mathrm{d} r_{\mathrm{s}}} u=0
$$

For our model, the hydrogen concentration $\phi$ will be assumed to be independent on the degree of ribbon bending. Solution of Eq. (17) gives the following expression for the mean radius of curvature $r_{s}$

$$
r_{s}=\frac{l_{s}}{l_{0}} \frac{L^{2}}{12 K} \frac{1}{\frac{L}{2}\langle\phi\rangle-\langle\phi x\rangle} .
$$

Using Eqs. $(1,6,10)$, the fraction $l_{s} / l_{0}$ can be expressed via $\langle\phi\rangle$

$\frac{l_{s}}{l_{0}}=1+K\langle\phi\rangle$.

Finally, the middle radius of curvature for hydrogenated bent ribbon is

$r_{s}=\frac{L^{2}}{12 K} \frac{1+K\langle\phi\rangle}{\frac{L}{2}\langle\phi\rangle-\langle\phi x\rangle} \approx \frac{L^{2}}{12 K} \frac{1}{\frac{L}{2}\langle\phi\rangle-\langle\phi x\rangle}$,

since $K\langle\phi\rangle \ll<1$.

A more appropriate parameter for analytic description of the curvature of a hydrogenated sample is the mean curvature $C_{s}$ of a sample

$C_{s}=\frac{1}{r_{s}} \approx \frac{12 K}{L^{2}}\left(\frac{L}{2}\langle\phi\rangle-\langle\phi x\rangle\right)$

In the following section expression for the curvature as a function of time will be obtained for the processes of hydrogenation and dehydrogenation, respectively.

\subsection{Sample curvature during hydrogenation}

Hydrogen diffusion, i.e. concentration of hydrogen inside the sample as function of time was recently studied in [6]. For the process of one side hydrogenation the concentration can be expressed as [6]

$$
\phi(x, t)=\phi_{0}-\phi_{0} \frac{x}{L}-\frac{2 \phi_{0}}{\pi} \sum_{n=1}^{\infty} \frac{\sin \left(\frac{n \pi}{L} x\right)}{n} \mathrm{e}^{-\frac{n^{2} \pi^{2} D}{L^{2}} t} .
$$

Using Eq. (22) average values in Eq. (21) can be calculated and the curvature of sample during the process of hydrogenation is expressed by the function

$$
C_{s}=\frac{K \phi_{0}}{L}\left(1-\frac{6}{\pi^{2}} \sum_{l=1}^{\infty} \frac{\mathrm{e}^{-\frac{(2 l)^{2} \pi^{2} D}{L^{2}} t}}{l^{2}}\right) .
$$

\subsection{Sample curvature during hydrogenation}

For the process of subsequent dehydrogenation from both sides of the ribbon the concentration can be expressed as [6]

$\phi(x, t)=\frac{2 \phi_{0}}{\pi} \sum_{n=1}^{\infty} \frac{\sin \left(\frac{n \pi}{L} x\right)}{n}\left(1-\mathrm{e}^{-\frac{n^{2} \pi^{2} D}{L^{2}} t_{0}}\right) \mathrm{e}^{-\frac{n^{2} \pi^{2} D}{L^{2}} t}$, 
where $t_{0}$ is the time of hydrogenation. And again, using Eq. (24) average values in Eq. (21) can be obtained and the curvature of sample during the process of dehydrogenation is expressed by function

$$
C_{s}=\frac{K \phi_{0}}{L} \frac{6}{\pi^{2}} \sum_{l=1}^{\infty}\left(1-\mathrm{e}^{-\frac{(2 l)^{2} \pi^{2} D}{L^{2}} t_{0}}\right) \frac{\mathrm{e}^{-\frac{(2 l)^{2} \pi^{2} D}{L^{2}} t}}{l^{2}}
$$

\section{RESULTS AND DISCUSSION}

The studied $\mathrm{Fe}_{85} \mathrm{~B}_{15}$ amorphous ribbon was prepared by rapid quenching from the melt in KFKI MTA in Budapest. This alloy does not exhibit any affinity to hydrogen. The length of sample used in experiments was $104 \mathrm{~mm}$, the width $w=11.8 \mathrm{~mm}$, thickness $L=0.029 \mathrm{~mm}$. Atomic hydrogen was introduced into the sample electrolytically from air side of the ribbon [6]. The duration of hydrogenation was $t_{0}=2$ hours.

The ribbon bending during the process of spontaneous dehydrogenation was recorded every 15 minutes (see Figs. $1,4)$.

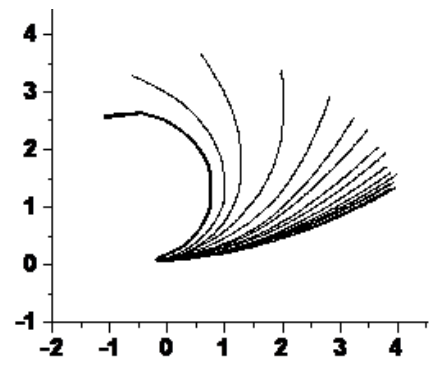

Fig. 4 Records of ribbon shape processed graphically which were used for the calculation of mean curvature as a function of time [7]. The curves from the left to the right were recorded

every 15 minutes. The scale on both axes is centimetre.

Using these records experimental dependence of the sample curvature as function of the time (concentration) was obtained [7]. Experimentally obtained values and corresponding fitted curve (Eq. (26)) are shown in Fig. 5

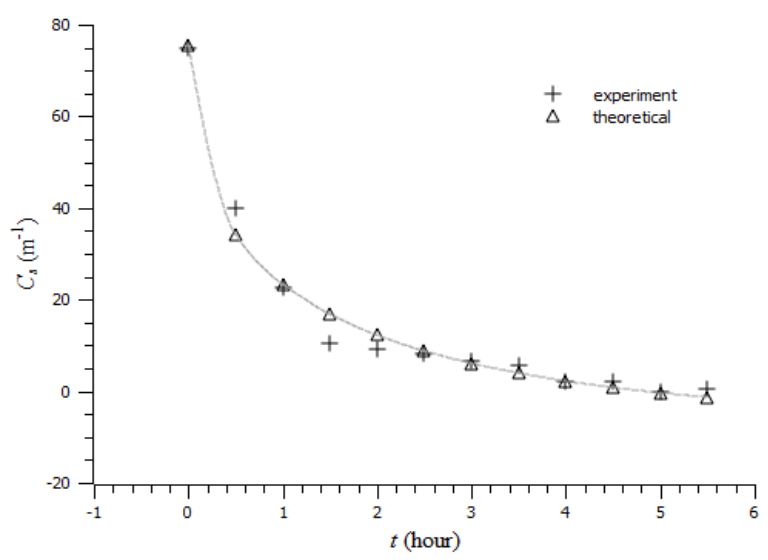

Fig. 5 Mean curvature $C_{s}$ of $\mathrm{Fe}_{85} \mathrm{~B}_{15}$ amorphous ribbon during process of dehydrogenation.

The known values [6] of concentration $\phi_{0}=2160 \mathrm{ppm}$ and diffusion coefficient $D=2.3 \cdot 10^{-15} \mathrm{~m}^{2} \mathrm{~s}^{-1}$ were used in the fitting procedure. As a result of this procedure the parameter $K$ introduced in Eq. 7 was obtained:
$K \phi_{0}=0.0033 \rightarrow K \approx 0.016(\mathrm{~nm})^{3}$. This parameter gives information about an increase of the volume caused by the presence of hydrogen atom. The value obtained approximately corresponds in all three dimensions to the so called Van der Waals radius for hydrogen (120 pm).

\section{CONCLUSION}

The proposed simple theoretical model for curvature of the one side hydrogenated thin ribbon from material without an affinity to hydrogen is in a good agreement with experimental results. Its fitting to experimental data provides important information on the local space enlargement around introduced hydrogen atom, which can change local magnetic characteristics.

Proposed method also provides information on the distribution of internal stresses depending on the distribution of hydrogen concentration in the sample. Since the studied materials exhibit high magnetostriction, the obtained theoretical results can be the base for further study of such important magnetic characteristics of the studied materials as e.g. magnetoelastic anisotropy constant [8].

\section{ACKNOWLEDGMENT}

This paper was developed within the project "Centre of Excellence for Integrated Research \& Exploitation of Advanced Materials and Technologies in Automotive Electronics" ITMS 26220120055.

\section{REFERENCES}

[1] SALIMON, A. I. - ASHBY, M. F. - BRECHET, Y. - GREER, A. L.: Mater. Sci. Eng., 375-377 (2004) 385.

[2] ALCALÁ, E. M. - LÓPEZ, E. - AROCA, C. SÁNCHEZ, M.C. - SÁNCHEZ, P.: Eur. Phys. J. B, 14 (2000) 473.

[3] ELIAZ, N. - ELIEZER, D.: Advanced Performance Materials, 6 (1999) 5.

[4] AKIHISA. I.: Acta. Mater., 48 (2000) 279.

[5] SPIVAK, L. V. - SKRYABINA, N. Ye.: International Journal of Hydrogen Energy, 24 (1999) 795.

[6] NOVÁK, L. - ZIMAN, J. - KOVALAKOVÁ, M. KLADIVOVÁ, M.: Acta Electrotechnica et Informatica, 12 (2012) 72.

[7] KOVAL'AKOVÁ, M. - NOVÁK, L. - ŠEVČOVIČ, L.: Proceedings of the 10th International Workshop on Applied Physics of Condensed Matter. , Slovak University of Technology, (2004) 138.

[8] KOVALAKOVÁ, M. - KOLLÁR, P. - NOVÁK, L. - ŠEVČOVIČ, L.: J. Magn. Magn. Mater., 304 (2006) e648.

Received January 14, 2013, accepted February 6, 2013 


\section{BIOGRAPHIES}

Mária Kladivová graduated in mathematical physics in 1980. In 2008 she received the PhD. degree from Pavol Jozef Šafárik University in Košice. She has been working at the Department of Physics, Faculty of Electrical Engineering and Informatics, Technical University of Košice since 1995. Study of ferromagnetic materials is a dominant field of her current research interest.

Ján Ziman graduated in solid state physics from Pavol Jozef Šafárik University in Košice. In 1994 he received the CSc. (PhD) degree from P.J. Šafárik University. He has been working at the Department of Physics, Faculty of Electrical Engineering and Informatics, Technical University Košice since 1983. Experimental study of ferromagnetic materials is a dominant field of his research work.
Ladislav Novák was born in 1948. In 1971 he graduated (MSc) from the Faculty of Science, University of P. J. Šafárik in Košice. He defended his CSc in 1984 and he became associate professor in 2007. Since 1998 he has been working at the Department of Physics, Faculty of Electrical Engineering and Informatics, Technical University in Košice. His research deals with magnetic properties of amorphous materials prepared by rapid quenching of the melt.

Mária Koval'aková was born in 1958. In 1982 she graduated from the Faculty of Science, Pavol Jozef Šafárik University in Košice. In 1999 she obtained her PhD degree at the Katholieke Universiteit in Leuven, Belgium. Since 1982 she has been working at the Department of Physics, Faculty of Electrical Engineering and Informatics, Technical University in Košice. Her scientific research includes study of ferromagnetic materials and NMR study of zeolites and related materials. 